\title{
Discussion on the Benefits of Port and Shipping Enterprises Based on Game Theory
}

\section{Mao' and ZHANG Shibin ${ }^{2}$}

${ }^{1}$ Logistics Research Center, ${ }^{2}$ Department of Mathematics, Shanghai Maritime University, Shanghai 201306, China

\begin{abstract}
In the field of port and shipping, the mutual interest relationship between the port and the shipping company has always been a hot topic.On the one hand, the port needs the shipping company to park its port operations to obtain economic benefits and social benefits.On the other hand, shipping companies have their own considerations when facing different types of ports, and must consider them from the perspectives of maximizing their own interests and optimizing service levels.In this paper, the Stackelberg model and the Nash equilibrium model in game theory are used to analyze the basis of the choice of the interests of the port and shipping enterprises to establish a multi-influence factor and multi-targeted model of the profit distribution of the port and shipping enterprises.According to the research in this paper, the appropriate cooperation between the two parties can further enhance the efforts of the port and shipping companies, and realize the rational distribution of interests between the port and the shipping company, so as to achieve a win-win situation in the entire port supply chain.
\end{abstract}

Keywords: Stackelberg model; Nash equilibrium; game theory; port and shipping enterprise
*Correspondence to Author:

LI Mao' and ZHANG Shibin ${ }^{2}$

${ }^{1}$ Logistics Research Center, ${ }^{2} \mathrm{De}$ partment of Mathematics, Shanghai Maritime University, Shanghai 201306, China

How to cite this article:

LI Mao and ZHANG Shibin. Discussion on the Benefits of Port and Shipping Enterprises Based on Game Theory. American Journal of Educational Research and $\mathrm{Re}$ views, 2019, 4:57.

\section{eScî̀Pub}

eSciPub LLC, Houston, TX USA. Website: http://escipub.com/ 


\section{Introduction}

With the gradual improvement of the level of economic globalization, the global maritime industry has also flourished, coupled with the evolution of supply chain thinking, in this era of the port supply chain came into being. As a combination of multimodal transport, the port integrates logistics information from land transport companies, shipping companies, suppliers and other participants. In the port supply chain, the port is the core part of it. Through the ship company, cargo owner and other participants, it is connected with suppliers and consumers around the world, so that the information flow and network flow are coordinated and optimized to form a set. Service value port supply chain with multiple modes of transportation and logistics ${ }^{[1]}$.

The continued steady development of the port lies in the effective cooperation of the various parties in the port supply chain. At this point, many scholars have done a lot of analysis and research. Chul-hwan $\mathrm{HAN}^{[2]}$ The factor analysis and regression methods are used to study the Busan container port, and the impact of the cooperation of the port supply chain participants on the overall port performance is determined. The results show that the port customer integration has an important impact on the overall quality performance, while the service level and price The strategy also has a positive impact on the throughput of the shipping company and the port itself. Sun Hui ${ }^{[3]}$ through the establishment of the game theory model, and then the game analysis of the vertical alliance of the port and shipping, and then through the port and shipping company strategic alliance interest distribution model, calculate the Shapley value for distribution, and finally can be concluded that the port and shipping companies Cooperation can maintain a dominant position in the competition, and this will become an inevitable choice for the future development of port and shipping enterprises.Yu Guangying ${ }^{[4]}$ applied game theory to enterprise supply chain cooperation, and made a corresponding analysis of the mutual game between leading companies and subordinate enterprises in the supply chain.Qi Xiuqing ${ }^{[5]}$ analyzed the four key factors of the distribution of the interests of the participants in the port supply chain, namely, input, risk, contribution and status. By establishing the port supply chain benefit distribution model, the analytic hierarchy process and the weighted summation method were used. The distribution of the benefits of each participant shows that this method is beneficial to the participants in the port supply chain to achieve a win-win situation. Zhou Lili[6] analyzed the factors affecting the distribution of the logistics service supply chain in the port and shipping logistics service supply chain, and combined the AHP method and the modified Shapley value method to make the port logistics service supply chain The distribution of benefits is more scientific and fair. Dong Yanmei ${ }^{[7]}$ based on the development model of port supply chain, taking Jiangsu coastal port logistics development as an example, and designing from effective participants, information services, organizational structure, etc., based on "integrated dynamic alliance" Port supply chain model. Wang Sheng ${ }^{[8]}$ through the establishment of the port and shipping supply chain optimization model and the use of PSO algorithm to verify the supply chain optimization of the port of Shandong Province, and finally proposed that can indeed improve the operational efficiency of the supply chain.

In order to simplify the concept, in the actual situation, the effective participants of the port 
are roughly divided into two types: the first is the cargo owner. When making the choice, they mainly consider whether the port can properly handle the goods within the set time limit, and the overall transportation cost. Is it cheap? The second is the shipping company. The main considerations include port handling efficiency, vessel turnover time, cabinet changing capacity, container handling fee, and convenience of storage and distribution. As the size of shipping companies continues to expand, port options are increasingly concentrated in shipping companies rather than shippers. The factors considered by the general shippers and shipping companies for port selection are: full transportation costs, full transit time, efficiency and reliability of customs clearance and related procedures, and "one-stop" port logistics services based on supply chain. Some scholars have also conducted research on ports and shipping companies. For example, Zhang Qi[9] discussed that the port side actively considers the shipping company's route rules in order to maximize its own interests in the context of market competition, and finally solves by Nash equilibrium. The port location of the shipping company and its own pricing strategy. Wu Jing ${ }^{[10]}$ compares the interests of ports and shipping companies in the absence of integration and mutual cooperation from the perspective of game theory, and draws the respective interests of port and shipping enterprises. It also analyzes the port and shipping enterprises in the game between the two sides. Potential problems. In this paper, it is also mainly to study the cooperative interest relationship between shipping companies and ports. There will be a relationship between the shipping company and the port in a certain period of time, such as information sharing, risk sharing, and mutual benefit. Therefore, research on supply chain coordination centered on port and shipping enterprises can not only has a certain essential understanding of the interests of both parties. More importantly, it can reduce time and capital costs, thereby improve the efficiency and competitive of the entire port supply chain.

\section{Description}

In the relationship between port and shipping enterprises, the mutual game between the shipping company and the port can be regarded as a Stackelberg model. In game theory, the Stackelberg game model is a very classic theory, which mainly highlights the dynamic game between the two sides in the context of complete information. Different from the Nash equilibrium model, in the Stackelberg model, the participating parties are not in an equal position for simultaneous decision-making, but have a dominant position and subordinate status. The dominant participant first makes the decision, and then the subordinate participant makes the decision based on the behavior of the dominant participant. On the other hand, because this is a dynamic game, the dominant players can improve and improve their previous action choices according to the decisions of the subordinate participants [11].

In the port supply chain, the specific realization is: under certain market capacity demand, the shipping company has a large autonomy to choose the number of ships, time, and quantity of goods to be docked at the port, so this paper assumes that the ship's side plays a leading role in the port and shipping enterprises. status. On the basis of establishing long-term cooperation between the shipping company and the port, the shipping companies are in a dominant position in the process of the game because of the priority decision, and the port is subordinate. At 
this time, the shipping company will cooperate with the port from the perspective of the port supply chain, and will also supervise and manage the service level of the port. The shipping company will provide relevant service guidance and construction of loading and unloading facilities for the port by investing a certain amount of effort into the port ${ }^{[12]}$.

In this paper, a port supply chain consisting of a single shipping company and a single port is assumed, and other factors are considered constant. The shipping company decided to park the port and the carrying capacity of the cargo, so the factors affecting the shipping company's choice of port are the price level and service level of the port. In the actual situation, the transaction price between the two is determined by the port side, and the transaction price sometimes involves factors such as the quantity of goods carried, so the transaction price is set as a non-decision variable. Secondly, the service level is mainly reflected in the level and extent of the efforts of the port side. The service level mainly refers to the shipping company carrying the goods to the port, and the port carries out a series of services such as inbound and outbound, loading and unloading and handling ${ }^{[13]}$.If the port party's service quality is high, the shipping company will give priority to the port in the future, and the number of goods carried will gradually increase, and the port side's throughput will be qualitatively improved. The interests of the shipping company mainly

$$
\pi_{a}=\alpha \vartheta a^{m} b^{1-m}-\frac{1}{2} p a^{2}-\frac{1}{2} \varepsilon p b^{2}
$$

Port profit function:

$$
\pi_{b}=\beta \vartheta a^{m} b^{1-m}-\frac{1}{2} p b^{2}+\frac{1}{2} \varepsilon p b^{2}
$$

For the first-order partial derivative of (2) for b: depend on its own efforts and the cost of hard work at the port.

(1) Assuming that the degree of effort of the shipping company is a, the degree of effort of the port is $b$, and the degree of effort of each of the two accounts for $m$ and $1-m$, and the influence of other factors is $\vartheta$, then The benefit function is $Q=\vartheta a^{m} b^{1-m}$;

(2) Assuming that the effort coefficient of the shipping company and the port is $p$, Then the effort costs of the shipping company and the port are $\frac{1}{2} \mathrm{pa}^{2}$ and $\frac{1}{2} \mathrm{pb}^{2}$;

(3) The shipping company and the port form a master-slave game, and the shipping company provides certain compensation for the service level of the port, assuming that the proportion of investment ise, Then the additional input cost of the shipping company is $\frac{1}{2} \varepsilon \mathrm{pb}^{2}$;

(4) Assume that the revenue coefficient of the shipping company and the port is respectively, $\alpha$ and $\beta$,among them $\alpha+\beta=1$;

(5) It is assumed that only the shipping company and the port share the profits of the entire port supply chain;

$$
\text { (6) } \alpha, \beta, a, b, \varepsilon, p, \pi \geq 0
$$

\section{Stackelberg model}

Shipping company's profit function: 


$$
\frac{\partial \pi_{b}}{\partial_{b}}=(1-m) \beta \vartheta a^{m} b^{-m}-(1-\varepsilon) p b
$$

Make $\frac{\partial \pi_{b}}{\partial_{b}}=0$, then $b^{*}=\left[\frac{(1-m) \beta \vartheta}{(1-\varepsilon) p}\right]^{\frac{1-m}{1+m}} a^{\frac{m}{1+m}}$

Will be $b^{*}$ substituted into (1):

$$
\pi_{a}=\alpha \vartheta a^{\frac{2 m}{m+1}}\left[\frac{(1-m) \beta \vartheta}{(1-\varepsilon) p}\right]^{\frac{1}{m+1}}-\frac{1}{2} p a^{2}-\frac{1}{2} \varepsilon p\left[\frac{(1-m) \beta \vartheta}{(1-\varepsilon) p}\right]^{\frac{2}{1+m}} a^{\frac{2 m}{m+1}}
$$

For (3) for a, find a first-order partial derivative:

$$
\frac{\partial \pi_{a}}{\partial_{a}}=\frac{2 m}{m+1} \alpha \vartheta a^{\frac{m-1}{m+1}}\left[\frac{(1-m) \beta \vartheta}{(1-\varepsilon) p}\right]^{\frac{1-m}{1+m}}-p a-\frac{m}{m+1} \varepsilon p\left[\frac{(1-m) \beta \vartheta}{(1-\varepsilon) p}\right]^{\frac{2}{1+m}} a^{\frac{m-1}{m+1}}
$$

Make $\frac{\partial \pi_{a}}{\partial_{a}}=0$, then $a^{*}=\frac{\vartheta}{p}(1-m)^{\frac{1-m}{2}}\left(\frac{m}{m+1}\right)^{\frac{m}{2}}\left[2 \alpha-(1-m) \frac{\varepsilon}{1-\varepsilon} \beta\right]^{\frac{m+1}{2}}\left(\frac{\beta}{1-\varepsilon}\right)^{\frac{1-m}{2}}$

$b^{*}=\frac{\vartheta}{p}(1-m)^{1-\frac{m}{2}}\left(\frac{m}{m+1}\right)^{\frac{m}{2}}\left[2 \alpha-(1-m) \frac{\varepsilon}{1-\varepsilon} \beta\right]^{\frac{m}{2}}\left(\frac{\beta}{1-\varepsilon}\right)^{1-\frac{m}{2}}$

It can be seen from the above formula that optimal profit of both parties and the total profit when the effort coefficient $p$ of the port and the of the port supply chain are obtained:

shipping company is larger, the enthusiasm of The optimal profit of the shipping company: each other is smaller, and at this time, the

$$
\pi_{a}^{*}=\frac{1}{2}\left[\frac{2 \alpha-(1-m) \frac{\varepsilon}{1-\varepsilon} \beta}{m+1}\right] \frac{\vartheta^{2}}{p}[(1-m) \beta]^{1-m}\left(\frac{m}{m+1}\right)^{m}\left(\frac{1}{1-\varepsilon}\right)^{1-m}\left[2 \alpha-(1-m) \frac{\varepsilon}{1-\varepsilon} \beta\right]^{m}
$$

The optimal profit of the port:

$$
\pi_{b}^{*}=\beta\left(\frac{1+m}{2}\right) \frac{\vartheta^{2}}{p}[(1-m) \beta]^{1-m}\left(\frac{m}{m+1}\right)^{m}\left(\frac{1}{1-\varepsilon}\right)^{1-m}\left[2 \alpha-(1-m) \frac{\varepsilon}{1-\varepsilon} \beta\right]^{m}
$$

In summary,the profit of the shipping company supply chain are consistent under the is related to $\varepsilon$, and the profit of the port has Stackelberg model, regardless of the proportion nothing to do with $\varepsilon$. This indicates that the profit of the shipping company's investment. of the port side and the profit of the entire port Total profit of the port supply chain:

$$
\pi^{*}=\vartheta a^{m} b^{1-m}=\frac{\vartheta^{2}}{p}[(1-m) \beta]^{1-m}\left(\frac{m}{m+1}\right)^{m}\left(\frac{1}{1-\varepsilon}\right)^{1-m}\left[2 \alpha-(1-m) \frac{\varepsilon}{1-\varepsilon} \beta\right]^{m}
$$

make $\left(\frac{1}{1-\varepsilon}\right)^{1-\mathrm{m}}\left[2 \alpha-(1-m) \frac{\varepsilon}{1-\varepsilon} \beta\right]^{m}=Y$

For $\mathrm{Y}$, the first-order partial derivative is obtained for $\varepsilon$ : 


$$
\begin{gathered}
\frac{\partial Y}{\partial_{\varepsilon}}=m\left[2 \alpha-(1-m) \frac{\varepsilon}{1-\varepsilon} \beta\right]^{m-1}\left[-(1-m) \frac{\beta}{(1-m)^{2}}\right]\left(\frac{1}{1-\varepsilon}\right)^{1-m} \\
+\left[2 \alpha-(1-m) \frac{\varepsilon}{1-\varepsilon} \beta\right]^{m}(1-m)\left(\frac{\beta}{1-\varepsilon}\right)^{-m} \frac{\beta}{(1-m)^{2}}
\end{gathered}
$$

Make $\frac{\partial Y}{\partial_{\varepsilon}}=0$, then $\varepsilon=1-\frac{1}{2 \frac{\alpha}{\beta}+(1-m)}$

It can be seen from the above formula that when the proportion of profit distribution between the shipping company and the port is larger, the value of $\frac{\alpha}{\beta}$ is larger, which means that the profit obtained by the shipping company as the dominant party in the entire port supply chain is greater than that of the port side, and the cost ratio invested by itself is $\varepsilon$. The bigger it is, the ship company is willing to help the port in order to get more benefits. The larger the value of $(1-m)$, the larger the value of $\varepsilon$, that is, the interest of the port in the entire port supply chain is increasing, and the service level of the port is becoming more and more prominent in the entire port supply chain, as long as the service level is obtained. Improve, then the shipping company is willing to invest the corresponding proportion of costs to help the port to improve service levels, so that both sides can maximize profits.

\section{Nsah Equilibrium model establishment}

Under the Nash equilibrium condition, it is assumed that the port and the shipping company make decisions according to their own optimal scheme, and the decision of the other party is not considered. The model of the port and shipping company is as follows:

The optimal profit model of the port:

$$
\max _{b}^{\prime}=\beta \vartheta a^{m} b^{1-m}-\frac{1}{2} p b^{2}
$$

Find the first-order partial derivative for $b$ :

$$
\frac{\partial \pi_{b}^{\prime}}{\partial_{b}}=(1-m) \beta \vartheta a^{m} b^{-m}-p b
$$

Make $\frac{\partial \pi_{b}^{\prime}}{\partial_{b}}=0$, then

$$
b^{* *}=\left[\frac{(1-m) \beta \vartheta}{p} a^{m}\right]^{\frac{1}{1+m}}
$$

The optimal profit model of the shipping company:

$$
\max _{a}^{\prime}=\alpha \vartheta a^{m} b^{1-m}-\frac{1}{2} p a^{2}=\alpha \vartheta\left[\frac{(1-m) \beta \vartheta}{p}\right]^{\frac{1-m}{1+m}} a^{\frac{2 m}{m+1}}-\frac{1}{2} p a^{2}
$$

Find the first-order partial derivative for a:

$$
\frac{\partial \pi_{a}^{\prime}}{\partial_{a}}=\frac{2 m}{m+1} \alpha \vartheta\left[\frac{(1-m) \beta \vartheta}{p}\right]^{\frac{1-m}{1+m}} a^{\frac{m-1}{m+1}}-p a
$$


Make $\frac{\partial \pi_{a}^{\prime}}{\partial_{a}}=0$, then $a^{* *}=\frac{\vartheta}{p}\left(\frac{m}{m+1}\right)^{\frac{m+1}{2}}(2 \alpha)^{\frac{m+1}{2}}[(1-m) \beta]^{\frac{1-m}{2}}$

$$
b^{* *}=\frac{\vartheta}{p}\left(\frac{m}{m+1}\right)^{\frac{m}{2}}(2 \alpha)^{\frac{m}{2}}[(1-m) \beta]^{1-\frac{m}{2}}
$$

The optimal profit of the port at this time is:

$$
\pi_{b}^{* *}=\left(\frac{1+m}{2} \beta\right) \frac{\vartheta^{2}}{p}[(1-m) \beta]^{1-m}\left(\frac{m}{m+1}\right)^{m}(2 \alpha)^{m}
$$

The optimal profit of the shipping company is:

$$
\pi_{a}^{* *}=\left(\frac{\alpha}{m+1}\right) \frac{\vartheta^{2}}{p}[(1-m) \beta]^{1-m}\left(\frac{m}{m+1}\right)^{m}(2 \alpha)^{m}
$$

The optimal profit of the entire port and shipping supply chain is:

$$
\pi^{* *}=\frac{\vartheta^{2}}{p}[(1-m) \beta]^{1-m}\left(\frac{m}{m+1}\right)^{m}(2 \alpha)^{m}
$$

\section{Nash Equilibrium and Stackelberg Model companies and ports}

Comparison Analysis

The optimal profit of the shipping company:

Optimal profit of two models of shipping

$$
\begin{aligned}
\pi_{a}^{* *}-\pi_{a}^{*}=\left(\frac{\alpha}{m+1}\right) & \frac{\vartheta^{2}}{p}[(1-m) \beta]^{1-m}\left(\frac{m}{m+1}\right)^{m}(2 \alpha)^{m} \\
& -\left\{\frac{\vartheta^{2}}{p}[(1-m) \beta]^{1-m}\left(\frac{m}{m+1}\right)^{m}\left(\frac{1}{1-\varepsilon}\right)^{1-m}\left[2 \alpha-(1-m) \frac{\varepsilon}{1-\varepsilon} \beta\right]^{m}\right\} \\
= & \frac{\vartheta^{2}}{p}[(1-m) \beta]^{1-m}\left(\frac{m}{m+1}\right)^{m}\left\{\frac{\alpha}{m+1}(2 \alpha)^{m}\right. \\
& \left.-\left[2 \alpha-(1-m) \frac{\varepsilon}{1-\varepsilon} \beta\right]^{m}\left(\frac{1}{1-\varepsilon}\right)^{1-m} \frac{\alpha-\frac{1}{2}(1-m) \frac{\varepsilon}{1-\varepsilon} \beta}{m+1}\right\} \\
= & \frac{\vartheta^{2}}{p}[(1-m) \beta]^{1-m}\left(\frac{m}{m+1}\right)^{m} \frac{\alpha}{m+1}(2 \alpha)^{m}\left\{1-\left\{\frac{1-\varepsilon\left\{\left[1+\frac{(1-m) \beta}{2 \alpha}\right]\right\}^{\frac{m+1}{2}}}{1-\varepsilon}\right\}\right.
\end{aligned}
$$

Therefore $\quad\left\{1-\left\{\frac{1-\varepsilon\left\{\left[1+\frac{(1-m) \beta}{2 \alpha}\right]\right\}^{\frac{m+1}{2}}}{1-\varepsilon}\right\}^{2}\right\}<0$, thus $\begin{aligned} & \text { The same is available, the optimal profit of the } \\ & \text { port: }\end{aligned}$ $\pi_{a}^{* *}-\pi_{a}^{*}<0$, indicate Stackelbergln the model,

the shipping company will get the best profit.

$$
\begin{aligned}
\pi_{b}^{* *}-\pi_{b}^{*}=\left(\frac{1+m}{2} \beta\right) \frac{\vartheta^{2}}{p}[(1-m) \beta]^{1-m}\left(\frac{m}{m+1}\right)^{m}(2 \alpha)^{m} \\
-\left\{\frac{\vartheta^{2}}{p}[(1-m) \beta]^{1-m}\left(\frac{m}{m+1}\right)^{m}\left(\frac{1}{1-\varepsilon}\right)^{1-m}\left[2 \alpha-(1-m) \frac{\varepsilon}{1-\varepsilon} \beta\right]^{m}\right\}<0
\end{aligned}
$$

https://escipub.com/american-journal-of-educational-research-and-reviews/ 
In the Stackelberg model, the port also Comparison of the optimal profit of the port and achieved the best profit. shipping supply chain under the two models

$$
\begin{gathered}
\pi^{* *}-\pi^{*}=\frac{\vartheta^{2}}{p}[(1-m) \beta]^{1-m}\left(\frac{m}{m+1}\right)^{m}(2 \alpha)^{m}-\left\{\frac{\vartheta^{2}}{p}[(1-m) \beta]^{1-m}\left(\frac{m}{m+1}\right)^{m}\left(\frac{1}{1-\varepsilon}\right)^{1-m}\left[2 \alpha-(1-m) \frac{\varepsilon}{1-\varepsilon} \beta\right]^{m}\right\} \\
=\frac{\vartheta^{2}}{p}[(1-m) \beta]^{1-m}\left(\frac{m}{m+1}\right)^{m}\left\{(2 \alpha)^{m}-\left[2 \alpha-(1-m) \frac{\varepsilon}{1-\varepsilon} \beta\right]^{m}\left(\frac{1}{1-\varepsilon}\right)^{1-m}\right\}
\end{gathered}
$$

Make $\mathrm{W}=\left[2 \alpha-(1-m) \frac{\varepsilon}{1-\varepsilon} \beta\right]^{m}\left(\frac{1}{1-\varepsilon}\right)^{1-m}=\left[1-\varepsilon\left(1+\frac{(1-m) \beta}{2 \alpha}\right)\right]^{m} \frac{(2 \alpha)^{m}}{1-\varepsilon}$

among them $1+\frac{(1-m) \beta}{2 \alpha}=1+\frac{1}{2} \frac{(1-m)}{\alpha}(1-\alpha)=1+\frac{1}{2}\left[\frac{1-m}{\alpha}-(1-m)\right] \geq 1$

So $1-\varepsilon\left(1+\frac{(1-m) \beta}{2 \alpha}\right) \leq 1,\left[1-\varepsilon\left(1+\frac{(1-m) \beta}{2 \alpha}\right)\right]^{m}>1-\varepsilon\left(1+\frac{(1-m) \beta}{2 \alpha}\right)>1-\varepsilon$

Then $\mathrm{W}>1, \pi^{* *}-\pi^{*} \leq 0$. It can be seen that in Comparison of efforts between two models of the Stackelberg model, the port and shipping shipping companies and ports supply chain will get the best profit.

The level of effort of the shipping company:

$$
\begin{gathered}
a^{* *}-a^{*}=\frac{\vartheta}{p}\left(\frac{m}{m+1}\right)^{\frac{m+1}{2}}(2 \alpha)^{\frac{m+1}{2}}[(1-m) \beta]^{\frac{1-m}{2}}-\left\{\frac{\vartheta}{p}(1-m)^{\frac{1-m}{2}}\left(\frac{m}{m+1}\right)^{\frac{m}{2}}\left[2 \alpha-(1-m) \frac{\varepsilon}{1-\varepsilon} \beta\right]^{\frac{m+1}{2}}\left(\frac{\beta}{1-\varepsilon}\right)^{\frac{1-m}{2}}\right\} \\
=\frac{\vartheta}{p}\left(\frac{m}{m+1}\right)^{\frac{m+1}{2}}[(1-m) \beta]^{\frac{1-m}{2}}\left\{(2 \alpha)^{\frac{m+1}{2}}-\left[2 \alpha-(1-m) \frac{\varepsilon}{1-\varepsilon} \beta\right]^{\frac{m+1}{2}}\left(\frac{1}{1-\varepsilon}\right)^{\frac{1-m}{2}}\right\}<0
\end{gathered}
$$

Port effort:

$$
b^{* *}-b^{*}=\frac{\vartheta}{p}\left(\frac{m}{m+1}\right)^{\frac{m}{2}}(2 \alpha)^{\frac{m}{2}}[(1-m) \beta]^{1-\frac{m}{2}}-\left\{\frac{\vartheta}{p}(1-m)^{1-\frac{m}{2}}\left(\frac{m}{m+1}\right)^{\frac{m}{2}}\left[2 \alpha-(1-m) \frac{\varepsilon}{1-\varepsilon} \beta\right]^{\frac{m}{2}}\left(\frac{\beta}{1-\varepsilon}\right)^{1-\frac{m}{2}}\right\}<0
$$

This shows that in the Stackelberg model, the port and shipping companies and changes in port and shipping companies have to work profits harder than the Nash equilibrium model. The relationship between the level of effort of Relationship between the level of hard work of the shipping company and the change in profit:

$$
\frac{\frac{\pi_{a}^{* *}-\pi_{a}^{*}}{\pi_{a}^{*}}}{\frac{a^{* *}-a^{*}}{a^{*}}}=\frac{\frac{\vartheta^{2}}{p}[(1-m) \beta]^{1-m}\left(\frac{m}{m+1}\right)^{m} \frac{\alpha}{m+1}\left\{(2 \alpha)^{m}-\left[2 \alpha-(1-m) \frac{\varepsilon}{1-\varepsilon} \beta\right]^{m+1}\left(\frac{1}{1-\varepsilon}\right)^{1-m}\right\}}{\frac{\vartheta}{p}\left(\frac{m}{m+1}\right)^{\frac{m+1}{2}}\{(1-m) \beta\}^{\frac{1-m}{2}}\left\{(2 \alpha)^{\frac{m+1}{2}}-\left[2 \alpha-(1-m) \frac{\varepsilon}{1-\varepsilon} \beta\right]^{\frac{m+1}{2}}\left(\frac{1}{1-\varepsilon}\right)^{\frac{1-m}{2}}\right\}}>1
$$

The result is greater than 1, which indicates that The relationship between the level of port effort the higher the level of effort of the shipping and the change in profits: company, the greater the profit. 


$$
\frac{\frac{\pi_{b}^{* *}-\pi_{b}^{*}}{\pi_{b}^{*}}}{\frac{b^{* *}-b^{*}}{b^{*}}}=\frac{\left(\frac{m+1}{2}\right) \beta \frac{\vartheta^{2}}{p}[(1-m) \beta]^{1-m}\left(\frac{m}{m+1}\right)^{m}\left\{(2 \alpha)^{m}-\left[2 \alpha-(1-m) \frac{\varepsilon}{1-\varepsilon} \beta\right]^{m}\left(\frac{1}{1-\varepsilon}\right)^{1-m}\right\}}{\frac{\vartheta}{p}\left(\frac{m}{m+1}\right)^{\frac{m}{2}}\{(1-m) \beta\}^{\frac{1-m}{2}}\left\{(2 \alpha)^{\frac{m}{2}}-\left[2 \alpha-(1-m) \frac{\varepsilon}{1-\varepsilon} \beta\right]^{\frac{m}{2}}\left(\frac{1}{1-\varepsilon}\right)^{1-\frac{m}{2}}\right\}}>1
$$

The result is greater than 1 , which indicates that The relationship between the level of joint the higher the port's efforts, the greater the efforts and the changes in the profit of the entire profits. port supply chain:

$$
\frac{\frac{\pi^{* *}-\pi^{*}}{\pi^{* *}}}{\frac{a^{* *}-a^{*}}{a^{* *}}}=\frac{\frac{\vartheta^{2}}{p}[(1-m) \beta]^{1-m}\left(\frac{m}{m+1}\right)^{m}\left\{(2 \alpha)^{m}-\left[2 \alpha-(1-m) \frac{\varepsilon}{1-\varepsilon} \beta\right]^{m}\left(\frac{1}{1-\varepsilon}\right)^{1-m}\right\}}{\frac{\vartheta}{p}\left(\frac{m}{m+1}\right)^{\frac{m+1}{2}}\{(1-m) \beta\}^{\frac{1-m}{2}}\left\{(2 \alpha)^{\frac{m+1}{2}}-\left[2 \alpha-(1-m) \frac{\varepsilon}{1-\varepsilon} \beta\right]^{\frac{m+1}{2}}\left(\frac{1}{1-\varepsilon}\right)^{\frac{1-m}{2}}\right\}}>1
$$

The result is greater than 1 , which indicates that company, the greater the profit of the entire port the higher the level of effort of the shipping supply chain.

$$
\frac{\frac{\pi^{* *}-\pi^{*}}{\pi^{* *}}}{\frac{b^{* *}-b^{*}}{b^{* *}}}=\frac{\frac{\vartheta^{2}}{p}[(1-m) \beta]^{1-m}\left(\frac{m}{m+1}\right)^{m}\left\{(2 \alpha)^{m}-\left[2 \alpha-(1-m) \frac{\varepsilon}{1-\varepsilon} \beta\right]^{m}\left(\frac{1}{1-\varepsilon}\right)^{1-m}\right\}}{\frac{\vartheta}{p}\left(\frac{m}{m+1}\right)^{\frac{m}{2}}\{(1-m) \beta\}^{\frac{1-m}{2}}\left\{(2 \alpha)^{\frac{m}{2}}-\left[2 \alpha-(1-m) \frac{\varepsilon}{1-\varepsilon} \beta\right]^{\frac{m}{2}}\left(\frac{1}{1-\varepsilon}\right)^{1-\frac{m}{2}}\right\}}>1
$$

The result is greater than 1 , which indicates that the higher the port's efforts, the greater the profit of the entire port supply chain.

In summary, for the port and shipping companies and the entire port supply chain, as long as they improve their efforts, the profits of each and the entire supply chain can be improved.Therefore, the shipping company and the port will choose the Stackelberg model for decision-making under the same influencing factors, that is, the shipping company is dominant and the port is subordinate, thus obtaining the entire port supply chain and their respective win-win situations.

\section{Conclusion}

In summary,under the Stackelberg model,both ports and shipping companies can maximize their own interests and maximize the benefits of the entire port supply chain.In this paper,it can also be concluded that if the port and shipping enterprises work hard to improve their own efforts (such as improving service levels, infrastructure construction, and technical guidance), it will help maximize the benefits of the entire port supply chain.It has encouraged the efforts of the port and shipping companies.Secondly,the benefit distribution mechanism in this paper reduces the investment of the shipping company to the port to some extent.However,in the face of conflicts of interest between the two parties,the port and shipping companies will adopt the Nash equilibrium model based on the principle of maximizing their own benefits. At the same time,this paper also considers the situation where the shipping company and the port provide services at the same time.According to the model provided by the shipping company and the full service provided by the port, it is clear that the port and shipping companies will choose to provide services and strengthen their efforts in order to optimize their own benefits.In short, the port supply chain has been more closely linked in terms of efficiency and information sharing in the context of the https://escipub.com/american-journal-of-educational-research-and-reviews/ 
Stackelberg model.In particular,the dominant shipping companies have deepened their mutual benefit cooperation with subordinate port companies.Thus, the relationship between the entire port supply chain has been further stabilized.

Author details: Li Mao (1993-), male,AnHui Maanshan, Master, the main research direction and operation of port operations

The second author: Zhang Shibin (1975-), male, Shandong Weifang, Professor, Ph.D., research direction for statistical analysis, statistics shipping, maritime logistics management calculated statistical methods.

\section{References}

[1]. Liu Meng, Yu Haihong. Summary of Research on Concept and Structure of Port Supply Chain[J].Technology and management,2015,17(01):63-67+80.

[2]. Chul-hwan HAN .Asian Journal of Shipping and Logistics, Volume 34,Issue 2, June 2018

[3]. Sun Hui.Research on the integration of port and shipping logistics based on vertical alliance [D].Ocean University of China, 2012.

[4]. Yu Guangying,Du Shaoqi.Discussion on Supply Chain Enterprise Cooperation Based on Game Theory[J].Statistics \& Decision,2013(21):49-52.

[5]. ZhangXiaomin.Research on Benefit Distribution of Port Supply Chain[J].Statistics \& Decision,2012(16):45-48.

[6]. Zhai Fangzhong, Zhou Lili,Jiang Baozhen. Research on Benefit Distribution in Port Logistics Service Supply Chain[J].China Water Transport (2nd Half), 2015, 15(3): 30-32.

[7]. DONG Yanmei.Design of Port Logistics Development Model Based on Supply Chain Management_-Taking Jiangsu Coastal Port as an Example[J].SAR Economics,2010(10):46-48.
[8]. Wang Sheng, Ren Xiaowei. Research on Port Supply Chain Optimization and Integration[J]. Ocean Development and Management, 2013, 30(07): 96-100.

[9]. Zhang Q, Wang $W$, Peng $Y$, et al. A game-theoretical model of port competition on intermodal network and pricing strategy[J]. Transportation Research Part E Logistics \& Transportation Review, 2018, 114:19-39.

[10]. Wu Jing. Research on issues related to port and shipping integration [D]. Shanghai Maritime University, 2007.

[11]. Zhang Yurui,Wang Hengshan, Yuan Xiuzhu.Application of Game Theory in Supply Chain Management-Status and Trend[J].Logistics Science and Technology,2008(11):115-116.

[12]. Yao Haofeng.Research on the coordination of inland rivers and ports based on price and service competition [D]. South China University of Technology, 2013.

[13]. Zhang Yali.Research on port group competitiveness based on collaborative competition [D]. Tianjin Polytechnic University, 2017.

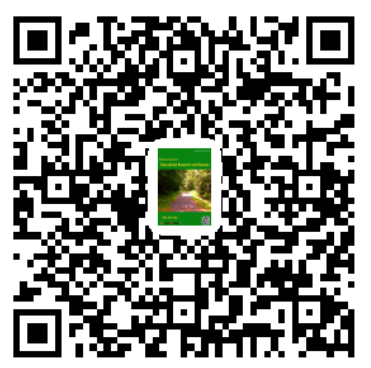

